\title{
Aplicación de una nueva terminología "BRUE: eventos breves, resueltos e inexplicados". Definiciones y recomendaciones
}

\author{
Application of a new terminology "BRUE: brief, resolved and unexplained events". \\ Definitions and recommendations
}

\author{
Daniel Zenteno Araos ${ }^{\mathrm{a}}$, Javiera Díaz Silva ${ }^{\mathrm{b}}$, Pablo Brockmann Veloso ${ }^{\mathrm{c}}$
}

aDepartamento de Pediatría, Universidad de Concepción. Servicio de Pediatría, Hospital Guillermo Grant Benavente, Concepción, Chile bInterna Medicina, Universidad de Concepción. Chile

`Departamento de Cardiología y Respiratorio Pediátrico, Centro del Sueño Pediátrico, Escuela de Medicina, Pontificia Universidad Católica de Chile

Recibido: 16 de octubre de 2018; Aceptado: 8 de septiembre de 2019

¿Qué se sabe del tema que trata este estudio?

Gran parte de la evidencia se basa en el término ALTE, que nos permitió mejorar el enfrentamiento de muchos pacientes. BRUE permite definir concretamente el episodio, determinar conductas según categorización de riesgo y optimizar el recurso en salud.
¿Qué aporta este estudio a lo ya conocido?

El artículo reúne la evidencia actual en relación con el tema, exponiendo las diferencias más relevantes para que el lector realice juicio propio de ambos términos y puedan incorporar el concepto de BRUE en su práctica clínica cotidiana.

\section{Resumen}

Hace tres décadas se propuso el término Apparent Life-Threatening Events (ALTE), siendo incorporado paulatinamente en el enfrentamiento clínico de estos pacientes; permitiendo determinar riesgos, atribuir causas y realizar tratamientos específicos. Sin embargo, llevó a realizar estudios y hospitalizaciones en muchas instancias considerados innecesarios, generando un aumento de los costos sanitarios. Por estos motivos nace el concepto de Brief Resolved Unexplained Events (BRUE), que pretende disminuir la subjetividad del evento y focalizar una estrategia de manejo según determinación del riesgo. En el siguiente artículo se analizan diferencias entre ALTE y BRUE según consensos internacionales y chilenos, profundizando en el enfrentamiento e incorporando consideraciones de relevancia para la práctica clínica cotidiana de lactantes que presentan un BRUE.
Palabras clave:

BRUE;

ALTE;

apneas;

muerte súbita 


\begin{abstract}
Three decades ago, the term Apparent Life-Threatening Events (ALTE) was proposed and was gradually incorporated into the clinical approach of these patients, allowing to determine risks, attribute causes, and perform specific treatments. However, this led to studies and hospitalizations considered unnecessary in many cases, increasing health costs. For this reason, the concept of Brief Resolved Unexplained Events (BRUE) was created, in order to reduce the subjectivity of the event and focus a management strategy according to the risk determination. This article analyzes the differences between ALTE and BRUE according to international and Chilean consensus, deepening the approach and incorporating relevant considerations for the daily clinical practice with infants who present a BRUE.
\end{abstract}

Keywords:

BRUE;

ALTE;

apnea;

sudden death

\section{Introducción}

En el año 1970, los conceptos de síndrome de muerte súbita del lactante y muerte súbita abortada comienzan a ser mencionados en la literatura, este último específicamente utilizado para referirse a aquellos pacientes que sufrían un evento aparentemente mortal pero que lograban vivir. Para similares presentaciones clínicas se empleaban los términos de muerte súbita frustra, crisis de apnea o crisis de cianosis, sin existir una categorización del riesgo que implicaba el episodio ${ }^{1}$.

En 1986 el Instituto Americano de Salud y Desarrollo Humano plantea el concepto de ALTE, del inglés "Apparent Life-Threatening Events", el cual paulatinamente se va incorporando en distintas instancias científicas internacionales hasta ser recomendado en 1994 por la Academia Americana de Pediatría (AAP). Luego de esto, se generó un notorio aumento de las investigaciones y publicaciones al respecto ${ }^{1}$. Entre los años 2003 y 2005, se publican los consensos europeo ${ }^{2}$ y latinoamericano de $\mathrm{ALTE}^{3}$. El año 2014, un grupo de especialistas de distintos centros asistenciales de nuestro país, publican el primer consenso de ALTE en Chile, conforme a la realidad de nuestro medio y a recomendaciones internacionales ${ }^{4}$.

Recientemente, en el 2016, el Subcomité de ALTE de la Sociedad Americana de Pediatría recomienda un nuevo concepto, en el marco de la publicación de una guía clínica para el enfrentamiento clínico de estos lactantes, donde sugiere modificar el término de ALTE por BRUE, del inglés Brief Resolved Unexplained Events, definido como eventos breves, resueltos e inexplicados, basándose en nuevas investigaciones, avances en medicina clínica y el análisis sobre el recurso en salud empleado ${ }^{5}$. Sin embargo, se limita a comparar los casos de ALTE menor con BRUE, dejando de lado a las situaciones de ALTE de riesgo mayor (figura 1).

El objetivo de este artículo es analizar las diferencias entre ALTE y BRUE según consensos internacio- nales y chilenos, profundizando en el enfrentamiento e incorporando consideraciones de relevancia para la práctica clínica cotidiana de lactantes que presentan un BRUE.

\section{Definiciones}

Las definiciones de ALTE y BRUE no son comparables mediante evidencias científicas, pues obedecen a recomendaciones de expertos en tiempos distintos y con gran variabilidad en el número de publicaciones. Hasta la fecha, existen muchas más publicaciones relacionadas con el concepto de ALTE que con el concepto de BRUE. En relación este último, los artículos principalmente reportan la incorporación del concepto y hay artículos sobre BRUE en los que se utilizó la misma definición de ALTE al reclutar los sujetos ${ }^{6}$.

La Academia Americana de Pediatría define ALTE como un evento brusco e inesperado que incluye manifestaciones de apnea junto con cambios de color y de tono muscular, donde el observador cree que el niño ha muerto ${ }^{1}$. El consenso chileno define el cuadro como un episodio brusco, que ocurre en un lactante menor de un año, con la presencia de 2 o más de las siguientes manifestaciones: apnea, cambio de color, cambio de tono o atoro; en el que es necesario de algún tipo de maniobra de reanimación y que para el observador genera la sensación de riesgo de vida para el menor ${ }^{4}$. Es decir, el consenso nacional incorpora la edad, determinación de número de características y la necesidad de maniobras de reanimación.

El concepto de BRUE corresponde a un evento que ocurre en menores de un año, presentado en forma brusca, corta (menos de 1 minuto) y resuelta, sin necesidad de reanimación. Incluye, uno o más de los siguientes criterios: cambio del patrón respiratorio (apnea, disminución o irregularidad), cambio del color (cianosis o palidez), alteración del tono muscular o de la conciencia y que, luego de una historia clínica y examen físico completo, no se encuentre explicación ${ }^{5}$. 
A diferencia de ALTE, el concepto BRUE considera la información obtenida en la anamnesis y procesada por un profesional de la salud calificado, incorpora la posibilidad de alteración de conciencia, excluye la rubicundez e involucra la alteración del patrón respiratorio como concepto más amplio que ALTE, alejándose del solo episodio de apnea. Adicionalmente, BRUE considera como límite de edad el año de vida, define un número mínimo de criterios, especifica las características de la coloración del paciente y amplia las posibles alteraciones respiratorias, siendo una definición globalmente más precisa y parecida a la de ALTE del consenso chileno ${ }^{7}$ (tabla 1 ). La figura 1 y muestran los aspectos relevantes sobre anamnesis y examen físico en la evaluación de lactantes (figuras 2 y 3 ).

\section{Motivos del cambio de concepto}

El concepto ALTE nos permitió mejorar procesos diagnósticos, categorizar a los pacientes por riesgo y por etiología atribuida. Progresivamente se fue reforzando la importancia de una anamnesis completa, de un examen físico adecuado e incluso a plantear algoritmos de estudios complementarios por etapas ${ }^{4,8}$. Sin embargo, generó un aumento en el gasto en salud, atribuidos principalmente a la indicación de estudios y hospitalizaciones, que en muchas ocasiones pudiesen haber sido innecesarias para determinar tratamientos específicos?.

El concepto cambia, considerando la necesidad de dar mayor precisión del evento y los aspectos relacionados con este, para posteriormente establecer el riesgo y asumir distintas estrategias de manejo. Adicionalmente, al introducir la apreciación del clínico y excluir la subjetividad del observador, nos podría permitir explicar de mejor forma aspectos clínicos y probablemente nos facilitaría una mayor calidad de investigación ${ }^{5,9}$.

Tieder J. y cols el 2008 publicaron un estudio que incluyó 20 hospitales con 12.067 pacientes que reveló que, a pesar de existir protocolos locales para ALTE, se practicaban diferentes tipos de exámenes y cantidad de ellos para cada caso, teniendo como consecuencia un alto gasto en recurso sanitario ${ }^{10}$. A nivel local, nuestro grupo presentó un estudio que reclutó casi 300 lactantes con diagnóstico de ALTE durante 7 años, comparando cohortes que fueron manejados con guías clínicas y otro con protocolo local más guía, demostrando que se lograba atribuir causas con mayor frecuencia cuando existía más estandarización en la evaluación clínica y además se redujo el tiempo de hospitalización ${ }^{11}$.

Altman RL. y cols el 2013, recopiló información de 40 años que incluyó 37 estudios y expone como principal factor de riesgo la prematuridad y la recurrencia de los episodios, junto con recomendar la realización de exámenes específicos sólo en casos de riesgo alto ${ }^{12}$.

\section{Categorización de riesgo en BRUE}

La guía clínica de BRUE de 2016 integra el antecedente de edad gestacional y de edad post-concepcional como un marcador relevante en la definición de paciente de bajo riesgo, incluyendo a todo aquel lactante mayor de 60 días o recién nacidos de pretérmino de 32 semanas en adelante con una edad gestacional corregida de 45 o más días, que no necesitaron reanimación cardiopulmonar por un profesional de salud, con un evento de menos de un minuto y que no presente recurrencia. Para estos pacientes la guía no recomienda hospitalización. Sin embargo, se sugiere considerar la observación en el recinto de urgencia por 1 a 4

y la posibilidad de realizarse algunos exámenes con resultados rápidos ${ }^{5}$.

La edad y la prematurez, son factores relevantes en estos lactantes, y punto fundamental para realizar la división entre BRUE de bajo o alto riesgo. Esto ha sido reportado en varios estudios. Tieder y cols, en una revisión sistemática, demostraron que a menor edad el riesgo de repetir eventos de ALTE durante la hospitalización es significativamente mayor en menores de 6 meses ${ }^{10}$. En esta línea, dos estudios chilenos de ALTE reportaron que la mayor parte de los casos sucede antes de los 6 meses, con un alza de los casos alrededor del $2^{\circ}$ mes de vida ${ }^{8,13}$. Otros autores, han demostrado que

Tabla 1. Diferencias entre definiciones de ALTE y BRUE según AAP

\begin{tabular}{l}
\hline ALTE (1987) \\
\hline Episodio brusco e inesperado, caracterizado por: \\
- Apnea (central u ocasionalmente obstructiva) \\
- Cambio de color (cianóticos o pálidos, en ocasiones eritematosa \\
- o pletórica) \\
- Asfixia on tono muscular (generalmente marcada flacidez) \\
- Observador cree que el niño ha muerto
\end{tabular}

\section{BRUE (2016)}

Lactante $<1$ año, con un episodio brusco, corto ( $<1$ minuto) y ahora resuelto, más $\geq 1$ de los siguientes criterios:

- Respiración irregular, disminución de la frecuencia respiratoria, o ausente $\rightarrow$ "cambio patrón"

- Cianosis o palidez

- Marcado cambio de tono (hipertonía o hipotonía)

- Nivel de conciencia alterado

- Sin explicación luego de anamnesis y examen físico

ALTE eventos de aparente amenaza a la vida del lactante. BRUE eventos breves, resueltos e inexplicados. 


\begin{tabular}{|l|l|l|}
\hline 1970 & \begin{tabular}{|l|l|}
\hline \\
$1986-1994$
\end{tabular} \\
\hline $2003-2005$ & $\begin{array}{l}\text { Síndrome de muerte súbita del lactante abortada o frustra, crisis de } \\
\text { apnea, crisis de cianosis. }\end{array}$ \\
\hline
\end{tabular}

Figura 1. Cronología ALTE y BRUE. ALTE: eventos de aparente amenaza a la vida de lactante. SOCHINEP: Sociedad Chilena Neumología Pediátrica. BRUE: eventos breves, resueltos e inexplicados.

\begin{tabular}{|c|c|c|c|c|}
\hline $\begin{array}{l}\text { RELATO DEL } \\
\text { EVENTO }\end{array}$ & ANTECEDENTES & $\begin{array}{l}\text { HISTORIA } \\
\text { FAMILIAR }\end{array}$ & $\begin{array}{l}\text { HISTORIA } \\
\text { SOCIAL }\end{array}$ & $\begin{array}{l}\text { ABUSO } \\
\text { INFANTIL }\end{array}$ \\
\hline $\begin{array}{l}\text { - Testigos, lugar } \\
\text { - Acciones, } \\
\text { posición y } \\
\text { movimientos } \\
\text { antes y durante el } \\
\text { evento } \\
\text { - Descripción de } \\
\text { conciencia, } \\
\text { coloración y } \\
\text { respiración } \\
\text { - Duración, } \\
\text { acciones de } \\
\text { testigos } \\
\text { - Estado general } \\
\text { del paciente luego } \\
\text { del evento }\end{array}$ & $\begin{array}{l}\text { - Historia perinatal } \\
\text { - Eventos similares } \\
\text { previos } \\
\text { - Reflujo } \\
\text { - Problemas } \\
\text { respiratorios } \\
\text { - Hospitalizaciones } \\
\text { o enfermedades } \\
\text { recientes } \\
\text { - Crecimiento y } \\
\text { desarrollo } \\
\text { - Inmunizaciones }\end{array}$ & $\begin{array}{l}\text { - Cuadros similares } \\
\text { en hermanos } \\
\text { - Muerte } \\
\text { inexplicada en } \\
\text { familiares de } 1^{\circ} \text { y } \\
2^{\circ} \text { grado } \\
\text { - Arritmias } \\
\text { - Enfermedades } \\
\text { genéticas o } \\
\text { metabólicas }\end{array}$ & $\begin{array}{l}\text { - Exposición a } \\
\text { tabaco o } \\
\text { contaminantes } \\
\text { - Exposición a } \\
\text { enfermedades } \\
\text { infecciosas } \\
\text { - Acceso a salud } \\
\text { - Adultos con } \\
\text { enfermedades } \\
\text { mentales o abuso } \\
\text { de sustancias } \\
\text { - Problemas o } \\
\text { estresantes } \\
\text { familiares }\end{array}$ & $\begin{array}{l}\text { - Situaciones } \\
\text { previas de } \\
\text { problemas legales } \\
\text { o de servicio } \\
\text { social } \\
\text { - Cambios en el } \\
\text { relato } \\
\text { - Incongruencias } \\
\text { entre historia y el } \\
\text { nivel de } \\
\text { desarrollo del } \\
\text { lactante }\end{array}$ \\
\hline
\end{tabular}

Figura 2. Aspectos principales en la anamnesis de un BRUE.

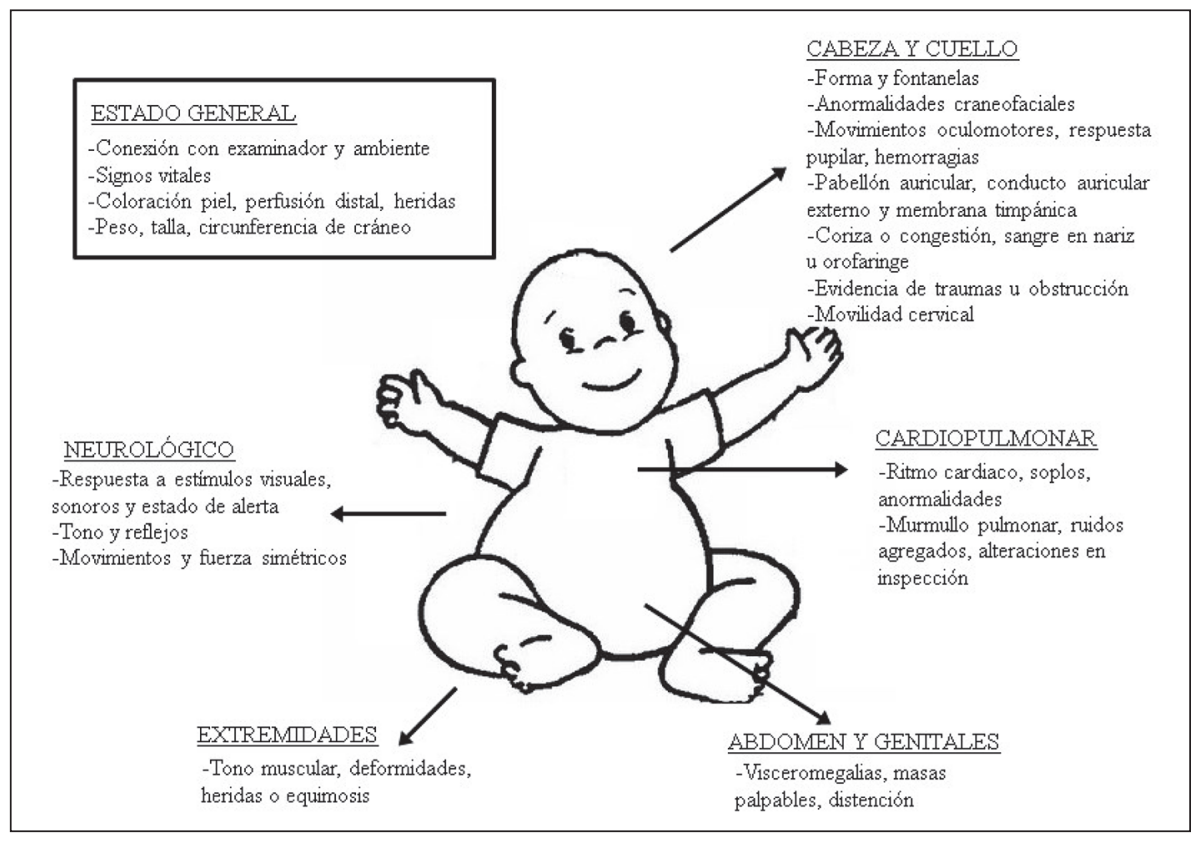

Figura 3. Aspectos principales en el examen físico de un BRUE. 
una edad gestacional corregida $<43$ semanas, aumenta hasta en 5,2 veces el riesgo de repetir un evento ${ }^{14}$.

La recurrencia de eventos durante o después de hospitalizaciones se ha relacionado con convulsiones o maltrato infantil ${ }^{12}$. Claudius evaluó el riesgo de múltiples ALTE, 24 horas previo a la hospitalización y encontró mayor probabilidad de recurrencia y gra$\operatorname{vedad}^{15}$.

La utilización de Reanimación Cardiopulmonar (RCP) es un criterio de gravedad e indicación de hospitalización en distintos consensos de manejo de ALTE, pese a la incerteza de su real necesidad. La guía de BRUE lo avala sólo cuando las maniobras RCP son realizadas por personal de salud entrenado ${ }^{2,3,16,17}$.

En un reporte de nuestro grupo constatamos que la principal causa de ALTE eran patologías respiratorias y que un $25 \%$ de los pacientes estudiados, egresaba del centro hospitalario sin causa evidente ${ }^{8}$. En cuanto a recurrencia, existen reportes que relacionan la presencia de síntomas respiratorios con riesgo de presentar nuevos episodios y ser reingresados a centros asistenciales de hasta 5 veces mayor que aquellos sin clínica respiratoria ${ }^{18}$. Por otro lado, pueden existir infecciones respiratorias que se manifiesten única o inicialmente como apneas, especialmente en lactantes menores ${ }^{20}$. Frente a estos datos epidemiológicos, los avances tecnológicos y la consideración sobre realizar estudios de resultados rápidos, se puede plantear realizar estudios etiológicos virales mediante técnicas disponibles en la actualidad.

\section{Recomendaciones para BRUE de bajo riesgo}

En el caso de BRUE de bajo riesgo no están indica- dos estudios neuroimagen, radiografías de tórax, exámenes de sangre, de orina; tampoco estudios de sueño, terapias antiepilépticas ni otros medicamentos. Estas conductas están respaldas por una serie de estudios que incluye la nueva guía de la $\mathrm{AAP}^{5}$.

La hospitalización no estaría indicada, situación reforzada recientemente con un metaanálisis que incluyo 12 artículos y 3.005 pacientes, donde se evaluó el riesgo de muerte post ALTE/ BRUE. Los autores concluyeron que el riesgo de muerte es similar al riesgo de un lactante que no presentó este evento ${ }^{6}$.

Puede considerarse la monitorización con oximetría de 1 a 4 horas en el centro asistencial tanto como la realización de electrocardiograma y estudios para descartar como casusa una infección por Bordetella pertussis ${ }^{5}$. Según el consenso americano, la monitorización durante 1 a 4 horas de signos vitales y oximetría es una posibilidad que podría plantearse, puesto que la recurrencia se presenta en un 12 y $16 \%$ de estos pacientes. En caso de presentarse nuevos episodios, es considerado un paciente de riesgo y tendría indicación de hospitalizar 5,8 .

Pese a la baja frecuencia de alteraciones cardiovasculares en el contexto de estos eventos, se puede identificar arritmias u orientar sobre alteraciones estructurales mediante un electrocardiograma, cuyo diagnóstico puede ser relevante para evitar eventos graves posteriores $^{21}$.

Se ha reportado infección por Bordetella Pertussis y ALTE, especialmente si existen factores epidemiológicos identificables en ausencia de inmunización a la madre y/o al lactante. Los estudios nasofaríngeos moleculares actuales proporcionan un diagnóstico rápido de la infección ${ }^{5,20,22}$ (tabla 2).

Tabla 2. Recomendaciones cardiopulmonares para BRUE de bajo riesgo y nivel de evidencia(5)

\begin{tabular}{|c|c|c|}
\hline & Recomendación & Calidad de la evidencia \\
\hline Hospitalización y monitorización & No & B \\
\hline Oximetría seriada y Observación (1-4h) & Alterativa & D \\
\hline Radiografía tórax & No & B \\
\hline Gases & No & B \\
\hline Polisomnografía & No & B \\
\hline Electrocardiograma & Alternativa & C \\
\hline Ecocardiograma & No & C \\
\hline Virus Respiratorios & No & C \\
\hline Bordetella pertussis & Alternativa & B \\
\hline
\end{tabular}




\section{Recomendaciones adicionales para el manejo de BRUE $^{5}$}

1. Educar sobre BRUE. Para esto la AAP proporciona un documento claro y conciso sobre los riesgos, cuando volver a consultar y su relación con la muerte súbita de lactante. Se encuentra disponible en español, por lo cual puede ser una herramienta de gran ayuda para nuestra práctica clínica.

2. Tomar decisiones compartidas con los padres. Es una recomendación que posee cierto grado de ambigüedad, pues abre las posibilidades a hospitalizaciones y estudios adicionales potencialmente innecesarios. Es de gran relevancia la experiencia del médico y del equipo de salud para orientar a los padres.

3. Entrenamiento a los cuidados sobre técnicas de reanimación cardiopulmonar. Puede ser complejo en un servicio de urgencia educar sobre RCP, tomando en cuenta la presión asistencial que poseen los equipos de salud en nuestro medio. Las condiciones de nuestro país no siempre poseen el mismo nivel de preparación en los diferentes niveles de atención que reciben estos pacientes. A juicio de los autores, podría plantearse educación de RCP básica a todos los padres de RN antes de su egreso hospitalario, con estrategias educativas escritas que permitan reforzar acciones en momentos de contingencia, como la presencia de un BRUE.

4. Educación sobre medidas de dormir seguro. Incluyen dormir en decúbito supino, evitar el colecho, ambiente libre de tabaco, uso de colchón firme y bien encajado, evitar el sobreabrigo, juguetes y almohadas en la cuna ${ }^{23}$. Si bien, no son una recomendación formal de las guías de BRUE referidas, debido a la relevancia epidemiológica que poseen, consideramos debe ser incorporado en las recomendaciones de estos pacientes, eventualmente mediante la utilización de tarjetas de cuna.

La dificultad se presenta en pacientes que presentan un evento más severo o que no cumplen los criterios de bajo riesgo definidos en la guía de BRUE. En estos casos, las recomendaciones de este nuevo concepto son insuficientes, y los protocolos locales pasan a ser determinantes para conductas de enfrentamiento ${ }^{4,5}$.

Los pacientes que no cumplen criterios de BRUE de bajo riesgo, se deberían hospitalizar por un mínimo de 24 horas en una unidad que permita el monitoreo cardiorrespiratorio continuo, para un estudio completo orientado en una anamnesis exhaustiva y posteriormente complementar con estudios escalonados según la realidad de cada centro de salud. Iniciar estudios con exámenes generales de sangre (hemograma, gases en sangre, glicemia, electrolitos plasmáticos, nitróge- no ureico), electroencefalograma, electrocardiograma, radiografía de tórax, y panel respiratorio. En caso de no tener hallazgos, continuar con evaluación por subespecialistas y estudios más complejos que procedan ${ }^{4,11}$ (figura 4).

El monitor cardiorrespiratorio domiciliario podría ser utilizado en pacientes de riesgo, luego completar su proceso de estudio y lograr estabilidad clínica. El equipo tratante es el encargado de evaluar caso a caso su indicación. Se recomienda el uso de monitores que permitan registrar movimiento cardiorrespiratorio, electrocardiográficos y que posean memoria para la validación de eventos. Es de relevancia mencionar que hasta la fecha no han demostrado disminuir el Síndrome de Muerte Súbita del Lactante ${ }^{4,24,25}$ (tabla 3).

\section{Estudio de sueño}

El conjunto de este tipo de estudios está indicado en pacientes catalogados de alto riesgo ${ }^{4}$. Si bien la polisomnografía es el estándar de oro para el diagnóstico de trastornos respiratorios del sueño, la poligrafía es una buena alternativa que puede realizarse en un paciente hospitalizado y monitorizado, siendo una ventaja en estos casos. Nuestro grupo publicó recientemente un artículo sobre poligrafía en menores de 3 meses: el 33\% correspondía a lactantes ingresados por ALTE mayor. El 93\% de los registros fueron confiables para su interpretación, similar a pacientes pediátricos mayores. El 16\% de los estudios eran anormales, los cuales presentaron principalmente un patrón de respiración inmadura $^{26}$. Si bien la poligrafía permite en la gran mayoría de los casos descartar apneas, existen casos en los cuales la polisomnografía completa es necesaria, especialmente en aquellos del ámbito neurológico.

En caso de no contar con estudios formales de sueño, la saturometría nocturna continua, podría ser una alternativa a considerar en menores de 3 meses. Este estudio tiene buena sensibilidad, pero baja especificidad, pudiendo ser utilizada como método de tamizaje. Sin embargo, si presenta alteraciones se requiere repetirlo o ejecutar otro estudio de sueño como una poligrafía ${ }^{27}$.

\section{BRUE en otros países}

En Europa hay grupos que han aceptado y publicado comentarios sobre la incorporación del concepto de BRUE. Se han referido a la ambigüedad de algunos criterios y la débil evidencia en las recomendaciones, sugiriendo la realización de hemoglucotest, la observación de 1 a 4 horas y una educación dirigida a los padres al momento de consultar ${ }^{28,29}$. 


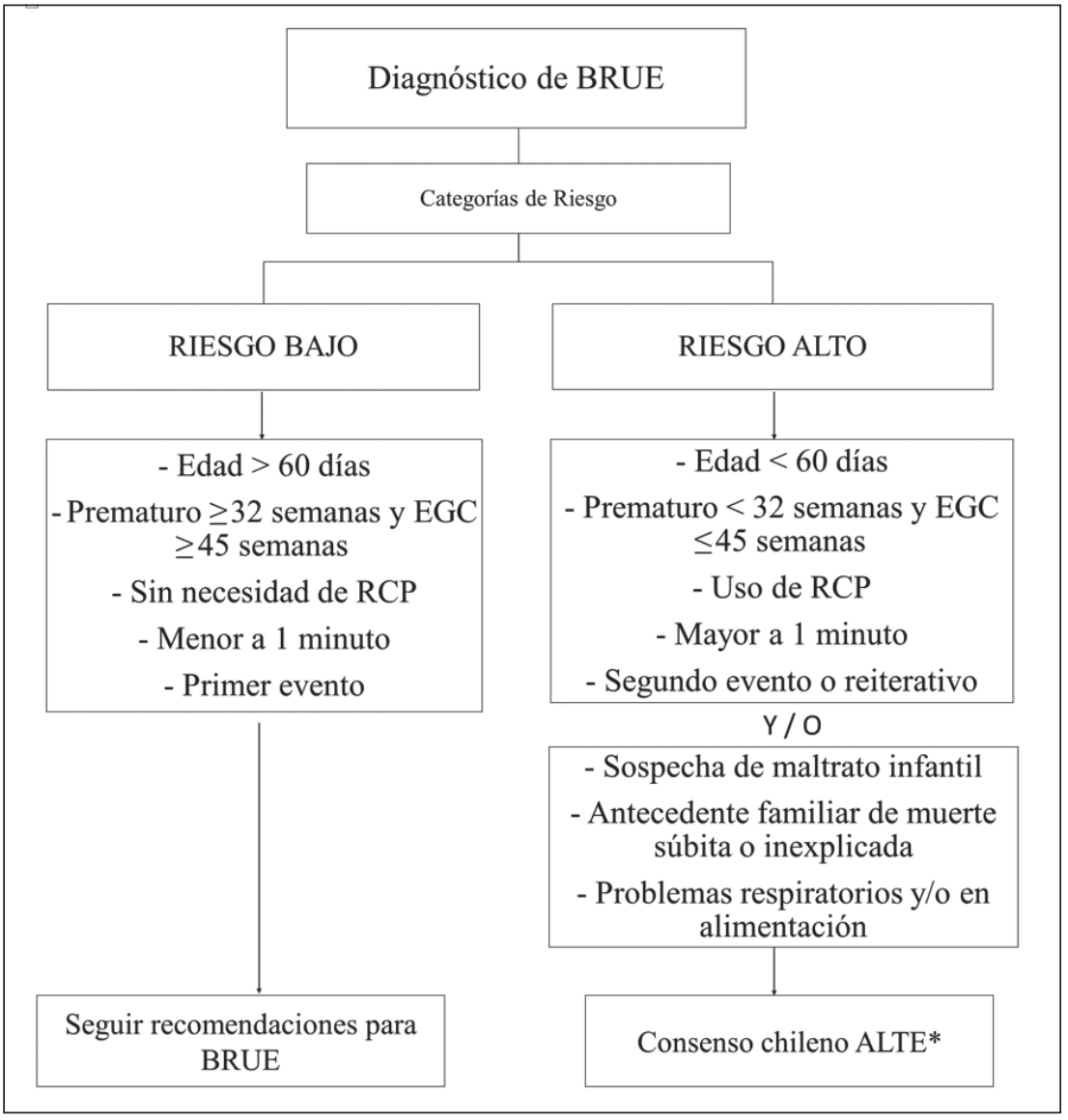

Figura 4. Caracterización de riesgo de paciente con sospecha de episodio de BRUE. * Se sugiere consenso chileno u otro algoritmo según realidad local.
Tabla 3. Indicaciones monitor cardiorrespiratorio domiciliario ${ }^{4}$

1. Causa idiopática: no se ha logrado llegar a una etiología evidente, pese a un estudio acucioso y polisomnografía

2. Episodios recurrentes ( $\geq 2$ episodios)

3. Episodio grave, sin causa clara, con reanimación formal o cianosis persistente

4. Episodio en menores con antecedentes de enfermedad de base (prematurez, displasia broncopulmonar, síndrome de Down, enfermedades neuromusculares, malformaciones craneofaciales, entre otros)

5. Episodios en pacientes que requieren de ventilación u oxígeno $\left(\mathrm{O}_{2}\right)$ domiciliario

6. Episodio en hermano de un fallecido por muerte súbita

\section{Conclusión}

En suma, BRUE es un término aceptado que se basa en un consenso que nos podría permitir optimizar el recurso en salud en nuestro medio. La anamnesis es el pilar diagnóstico para poder determinar el riesgo. Si el riesgo es bajo, se pueden seguir recomendaciones de la AAP planteados en la guía de BRUE y descritas en este artículo. Sin embargo, si el riesgo es alto, se deberían seguir protocolos locales, como el consenso chileno de ALTE. Resulta de relevancia incorporar las sugerencias dirigidas a los padres sobre educación en el concepto de BRUE y de RCP. Las recomendaciones planteadas por este nuevo concepto podrían ser incorporadas paulatinamente en los distintos centros asistenciales de nuestro país.

\section{Conflicto de intereses}

Los autores declaran no tener conflicto de intereses. 


\section{Referencias}

1. National Institutes of Health Consensus: Development Conference on Infantile Apnea and Home Monitoring. Consensus Statement. Pediatrics. 1987;79:292-9.

2. Kahn A. Recommended clinical evaluation of infants with an apparent life-threatening event. Consensus Document of the European Society for the Study and Prevention of Infant Death, 2003. Eur J Pediatr 2004;163:108-15.

3. Comité en síndrome de muerte súbita del lactante. Asociación Latinoamericana de Pediatría. Guía de práctica clínica 2005. Episodio de posible amenaza a la vidaALTE. Disponible en: https://alape.org/ wp-content/uploads/2018/08/consenso_ alte.pdf (Fecha acceso: 30 de agosto de 2019).

4. Brockmann P, Abara S, Campos C, et al. Consenso sobre el manejo de eventos de aparente amenaza a la vida del lactante (ALTE): Comisión de Sueño, Sociedad Chilena de Neumología Pediátrica 2013. Rev chil Pediatr. 2014; 85:378-87.

5. Tieder J, Bonkowsky J, Etzel R, et al. Brief Resolved Unexplained Events (Formerly Apparent Life-Threatening Events) and Evaluation of Lower-Risk Infants. Pediatrics. 2016;137-69.

6. Brand D, Fazzari M. Risk of Death in Infants Who Have Experienced a Brief Resolved Unexplained Event: A MetaAnalysis. J Pediatr. 2018;197:63-7.

7. Arane K, Claudius I, Goldman R. Brief resolved unexplained event: New diagnosis in infants. Canadian Family Physician. 2017;63:39-41.

8. Zenteno Daniel, Quiroz Gonzalo, Celis Magdalena, Tapia Jaime. Causas atribuidas a eventos de aparente amenaza a la vida del lactante. Rev Chil Pediatr. 2008; 79:163-71.

9. Rocca Manuel. BRUE: a new term and approach that could improve our practice. Arch Argent Pediatr. 2016; 114:506-7.

10. Tieder J, Cowan C, Garrison M, Christakis D. Variation in Inpatient Resource Utilization and Management of Apparent Life-Threatening Events. J Pediatr. 2008;152:629-35.
11. Zenteno D, Peña R, Fuentes C, Tapia J, Verbal D, Rodríguez I. Efecto de un enfoque estandarizado en lactantes hospitalizados por eventos de aparentemente amenaza a la vida. Biomédica. 2018; 38: 479-85.

12. Tieder J, Altman R, Bonkowsky J, et al. Management of Apparent LifeThreatening Events in Infants: A Systematic Review. J Pediatr 2013; 163:949.

13. Brockmann P, González X, Bertrand P, Sánchez I, Holmgren N. Perfil clínico de lactantes hospitalizados por un episodio de ALTE (Apparent Life Threatening Event). Rev Chil Pediatr 2006;77:267-73.

14. Al-Kindy H, Gélinas JF, Hatzakis G, Coté A. Risk factors for extreme events in infants hospitalized for apparent lifethreatening events. J Pediatr 2009; 154: 317-9.

15. Claudius I, Keens T. Do all infants with apparent life-threatening events need to be admitted? Pediatrics 2007;119:679-83.

16. Kaji AH, Claudius I, Santillanes G, et al. Apparent life-threatening event: multicenter prospective cohort study to develop a clinical decision rule for admission to the hospital. Ann Emerg Med. 2013; 61:379-87.

17. Samuels M, Poets C, Noyes J, Hartmann $\mathrm{H}$, Hervenston J, Southall D. Diagnosis and management after ALTE in infants and young children who received cardiopulmonary resuscitation. BMJ. 1993;306:489-92.

18. Ueda R, Nomura O, Maekawa T, Sakai $\mathrm{H}$, Nakagawa S, Ishiguro A. Independent risk factors for recurrence of apparent life-threatening events in infants. Eur J Pediatr. 2017;176:443-8.

19. Zenteno D, Peña R, Cerda M, Fuentes C. Apneas e infecciones en lactantes. Neumol Pediatr. 2012;7:48-50.

20. Hoki R, Bonkowsky JL, Minich LL, Srivastava R, Pinto NM. Cardiac testing and outcomes in infants after an apparent life-threatening event. Arch Dis Child. 2012;97:1034-8.

21. Centers for Disease Control and Prevention. Pertussis (whooping cough): diagnostic testing. (en línea). Agosto 2017.
(Fecha de acceso: 28 de agosto de 2019). Disponible en: https://www.cdc.gov/ pertussis/clinical/diagnostic-testing/index. html.

22. Carlin R, Moon R. Risk factors, Protective Factors, and Current Recommendations to Reduce Sudden Infant Death Syndrome: A Review. JAMA Pediatr. 2017;171:175-80.

23. Ueda R, Nomura O, Maekawa T, Sakai H, Nakagawa S, Ishiguro A. Independent risk factors for recurrence of apparent lifethreatening events in infants. Eur J Pediatr 2017;176:443-8.

24. Hunt CE, Corwin MJ, Lister G, et al. Precursors of cardiorespiratory events in infants detected by home memory monitor. Pediatr Pulmonol. 2008;43:8798.

25. Poets A, Urschitz MS, Steinfeldt R, Poets CF. Risk factors for early sudden deaths and severe apparent lifethreatening events. Archives of disease in childhood Fetal and neonatal edition 2012;97(6):395-7.

26. Zenteno D, Rodríguez-Núñez I, Molina I, et al. Poligrafía en menores de 3 meses hospitalizados. Rev Chil Pediatr. 2017;88:230-5.

27. Zenteno D, Bancalari A, Navarro X, Díaz V, Rodríguez-Núñez I, Brockmann P. Diagnóstico de los trastornos respiratorios del sueño en recién nacidos con sospecha de apneas: comparación entre la saturometría nocturna y la poligrafía. Rev Chil Pediatr. 2017;88:759-64.

28. Tate C, Sunley R. Brief resolved unexplained events (formerly apparent life-theathing events) and evaluation of lower - risk infants. Arch Dis Child Pract Ed. 2018;103:95-8.

29. Zafra M, Alonso de la Hoz J, Fernández B, Nieto N. Reciente modificación de la Academia Americana de Pediatría del concepto episodio aparentemente letal (ALTE) por el actual evento breve resuelto inexplicado (BRUE). Comentarios a propósito de una experiencia de cinco años en monitorización cardiorrespiratoria domiciliaria. Rev Pediatr Aten Primaria. 2017;19:23-8 